\title{
Facile preparation and performance study of antibacterial regenerated cellulose carbamate fiber based on $\mathbf{N}$-halamine
}

\author{
Jiewen Hu $\cdot$ Ruojia Li $\cdot$ Shaotong Zhu $\cdot$ Gangqiang Zhang $\mathbb{D} \cdot$ Ping Zhu
}

Received: 26 November 2020/ Accepted: 16 March 2021/Published online: 8 April 2021

(C) The Author(s), under exclusive licence to Springer Nature B.V. 2021

\begin{abstract}
With the outbreak of coronavirus disease (COVID-19) which has incalculable disasters and economic losses, people have given increasing attention to the health and safety of textile and fiber materials. In this study, an eco-friendly, facile, and cost-effective wet-spinning cellulose carbamate fiber technology was developed, and N-halamine regenerated cellulose fiber ( $\mathrm{RCC}-\mathrm{Cl})$ with rechargeable and rapid bactericidal properties were prepared by the Lewis acid-assisted chlorination method. The chemical properties of the fibers were characterized by Fourier transform infrared spectroscopy, X-ray diffraction, X-ray photoelectron spectroscopy, thermogravimetric analysis, and energy-dispersive X-ray spectroscopy. The mechanical and surface topography of the treated fiber was investigated by tensile testing and scanning electron microscopy. The results showed that the mechanical properties of RCC-Cl fibers can reach a breaking strength of $12.1 \mathrm{cN} /$ tex and a breaking elongation of $41.4 \%$ with the optimized spinning process. Furthermore, RCC-Cl showed
\end{abstract}

J. Hu · R. Li · S. Zhu · G. Zhang $(\varangle) \cdot$ P. Zhu ( $\varangle)$ College of Textile and Clothing, Institute of Functional Textiles and Advanced Materials, State Key Laboratory of Bio-Fibers and Eco-Textiles, Collaborative Innovation Center of Marine Biomass Fibers Materials and Textiles of Shandong Province, Qingdao University,

Qingdao 266071, China

e-mail: cris8866@163.com

P. Zhu

e-mail: pzhu99@163.com excellent antimicrobial activities, which can inactivate Escherichia coli and Staphylococcus aureus at a concentration of $10^{7} \mathrm{CFU} / \mathrm{mL}$ within $1 \mathrm{~min}$. This work provided a novel approach to produce regenerated cellulose fibers with antibacterial properties, showing great potential in the field of functional textiles.

Keywords Cellulose carbamate fiber $\cdot \mathrm{N}$-halamine · Antibacterial · Textile

\section{Introduction}

As the most abundant natural polymer on earth, cellulose has attracted much attention due to its renewability, wide availability, low cost, biocompatibility, and biodegradability (Basu et al. 2016; Coseri 2017; Taheri et al. 2020). Regenerated cellulose fiber has been widely used in the medical, textile, water treatment, automotive, and other industries due to its excellent properties (Liu et al. 2019; Okugawa et al. 2020; Sharma et al. 2019; Wu et al. 2018). Additionally, in the processing of traditional regenerated cellulose fibers (viscose fibers), the release of large amounts of $\mathrm{CS}_{2}$ and $\mathrm{H}_{2} \mathrm{~S}$ poses serious harm to the environment and human health (Yang et al. 2018). Therefore, it is meaningful to develop eco-friendly 
regenerated cellulose fibers to replace traditional fibers.

The green production process of cellulose carbamate fiber can maximize the use of equipment in traditional processes at a low cost making it expected to replace traditional processes (Fu et al. 2014a, 2015). Cellulose carbamate fiber has fascinating dyeing properties and excellent physicochemical properties (Fu et al. 2014a, b; Teng et al. 2018). In recent years, certain types of research have been conducted on the structure and properties of cellulose carbamate fibers.

Regenerated cellulose fibers, including cellulose carbamate fibers, lacking antibacterial properties has restricted their development and application in medical and health-related fields (Elsayed et al. 2020; Fu et al. 2014b; Zahra et al. 2020). In particular, with the outbreak of coronavirus disease (COVID-19) which caused incalculable disasters and economic losses, the antibacterial properties of regenerated fibers have received increasing attention (Desai and Aronoff 2020; He et al. 2020).

To cope with the threat of pathogenic microorganisms to human health and economy, the antibacterial properties conferred on cellulose fibers as the basic unit of textiles have aroused widespread research interest. The usual strategy for imparting antibacterial properties to fibers is to introduce $\mathrm{N}$-halamine (Wang et al. 2020), antibiotics (Bekri et al. 2020), nano silver (Guo et al. 2020), chitosan (Raza et al. 2020), and other antibacterial substances into fiber materials. In particular, N-halamine has become one of the most promising bio-protective material disinfectants due to its rapid inactivation rate, broad-spectrum biocidal activity, renewable biocidal activity, safety, and ecofriendly properties (Liu et al. 2018; Zhang et al. 2019a). Although the application potential of N-halamine in cellulose fibers is excellent, the main problem is that the $\mathrm{N}$-halamine precursors are mixed into the fiber, rather than reacting by covalent chemical bonds (Chen et al. 2016). This would cause the sterilizing effect to be reduced due to the loss of the doped sterilizing group (Sun et al. 2012). In addition, the $\mathrm{N}$-halamine cellulose fibers obtained by finishing modification also have the problems of impaired mechanical properties and complicated processes (Kocer et al. 2011; Li et al. 2013; Pan et al. 2018). Accordingly, the construction of uniform and compact, mechanically robust, and convenient process of
$\mathrm{N}$-halamine antibacterial cellulose fiber is still the main problem to be solved.

In this study, cellulose carbamate could be chlorinated into $\mathrm{N}$-halamine antibacterial products due to its amide group. Therefore, we have developed an ecofriendly, facile, and cost-effective wet-spinning cellulose carbamate fiber technology, and prepared $\mathrm{N}$-halamine regenerated cellulose fiber ( $\mathrm{RCC}-\mathrm{Cl}$ ) with rechargeable and rapid bactericidal properties by the Lewis acid-assisted chlorination method. The structure and properties of the fiber after chlorination were characterized by Fourier transform infrared spectroscopy (FTIR), X-ray diffraction (XRD), X-ray photoelectron spectroscopy (XPS), thermogravimetric analysis (TGA), scanning electron microscopy (SEM), energy-dispersive X-ray (EDX) spectroscopy, and tensile tests. The effect of the process conditions on the mechanical properties and active chlorine content of RCC-Cl was studied. The antibacterial properties, rechargeability, and stability of $\mathrm{RCC}-\mathrm{Cl}$ were evaluated.

\section{Material and experiment}

\section{Material}

Cellulose pulp with an $\alpha$-cellulose content of $92 \%$ was provided by Shandong Yinying Chemical Fiber Co., Ltd. (Shandong, China), which were degraded in $18 \mathrm{wt} \% \mathrm{NaOH}$ at $80^{\circ} \mathrm{C}$ for $2 \mathrm{~h}$ before further treatment. Wash with water to neutral, the alkalitreated cellulose was undergone a $24 \mathrm{~h}$ drying process at $80{ }^{\circ} \mathrm{C}$ and is referred to as Cel. Sodium hydroxide was obtained from Xilong Chemical Co., Ltd. N, N-Dimethylacetamide (DMAC) was purchased from Tianjin Fuyu Fine Chemical Co., Ltd. S. aureus (ATCC 6538) and E. coli (ATCC 25922) were provided by the China Depositary Biotechnology Center. Sodium thiosulfate (0.1 M solution in water) and phosphate buffered solution (PBS) were provided by J\&K Scientific Co., Ltd and Shanghai Macleans Biochemical Technology Co., Ltd, respectively. Other chemicals were from Sinopharm Chemical Reagent Co., Ltd., and used without further purification. 
Preparation of regenerated antibacterial cellulose fiber

Cellulose pulp and urea were added to the threenecked flask in a weight ratio of $1: 3(\mathrm{w} / \mathrm{w})$ and a certain amount of DMAC was added as a carrier. The mixture was heated to $160{ }^{\circ} \mathrm{C}$ in an oil bath, and the reaction was stirred for $6 \mathrm{~h}$ in a nitrogen atmosphere. The $\mathrm{NH}_{3}$ generated during the reaction was discharged through a rubber tube and was absorbed by water. At the end of the reaction, the mixture was cooled to room temperature. The mixture was filtered to obtain the product and washed thoroughly with distilled water to remove urea and DMAC remaining in the product. Finally, the cellulose carbamate (CC) was dried in an oven at $80{ }^{\circ} \mathrm{C}$ for $4 \mathrm{~h}$ for the subsequent experiment.

A mixed system solution of $8 \mathrm{wt} \%$ sodium hydroxide and $12 \mathrm{wt} \%$ urea was prepared in a beaker and placed in a cold well to cool to $-12.5^{\circ} \mathrm{C}$. A certain amount of $\mathrm{CC}$ was added and stirred for $5 \mathrm{~min}$ to obtain transparent $\mathrm{CC}$ solutions with different concentrations (3-7\%). After centrifugal defoaming, the spinning solution was poured into the reactor of the spinning machine. The spinneret was fabricated of 75 holes with diameter of $0.1 \mathrm{~mm}$. After forming in a sulfuric acid coagulation bath of different concentrations (3-12\%), nascent fibers with different degrees of drafting (1:1.1-1.4) were obtained. After the water bath, the salt and acid on the surface of the RCC were rinsed with deionized water, and then the RCC were soaked in deionized water for $1-2 \mathrm{~h}$ and washed to neutrality to ensure that the salt and acid attached to the fiber were removed. The RCC were put into a chlorination bath for chlorination treatment. After chlorination, the samples were soaked and washed with deionized water three times for 3 min each time, and RCC-Cl were obtained by natural drying (Fig. 1).

\section{Characterization}

\section{Chemical properties}

The infrared absorption spectra of cellulose pulp (Cel), CC, RCC, and RCC-Cl were measured with a Nicolet iS50 FTIR spectrometer in ATR mode. The scanning range was $400-4000 \mathrm{~cm}^{-1}$, the number of scans was 32 , and the data point interval was $1.2858 \mathrm{~cm}^{-1}$.
The nitrogen content (N\%) of $\mathrm{Cel}, \mathrm{CC}, \mathrm{RCC}$, and RCC-Cl was determined with a VARIO EL III elemental analyser (Elementar Co., Germany).

The D8 Advance X-ray diffractometer (Brooke Company) was used to obtain 3000 counts in the range of $5^{\circ}-35^{\circ}$ with a step size of $0.01^{\circ}$ to characterize the crystal structure of the samples. X-ray photoelectron spectroscopy (XPS) spectra were recorded by a ESCALAB 250Xi instrument (Thermo Scientific, USA) equipped with Al Ka excitation radiation (hv$1486.6 \mathrm{eV}$ ) in the range of- $10-1530 \mathrm{eV}$ to measure the chemical composition of samples.

TGA was performed using the synchronous thermal analyzer TG209F3 of NETZSCH Company with a heating speed of $10^{\circ} \mathrm{C} / \mathrm{min}$ from 25 to $800{ }^{\circ} \mathrm{C}$ under nitrogen flow.

\section{Physical properties}

The breaking strength and elongation at break of RCC and RCC-Cl were tested with a FAVIMAT AIROBOT Fiber Test electronic single fiber strength tester (Textechno, Germany). The fibers were tested under the conditions of an 8.5 dex linear density of regenerated cellulose fibers, $0.8 \mathrm{cN}$ pretension, $10 \mathrm{~mm}$ initial clamp distance, and $20 \mathrm{~mm} / \mathrm{min}$ tensile rate.

The surface morphology, thickness, and element distribution of cellulose membranes before and after chlorination were observed by SEM-EDX (TESCANVEGA3, USA; SERIAL\#: E1856-C2B, USA). The samples were sprayed with gold in a vacuum for $1 \mathrm{~min}$.

\section{Analytical titration}

The loaded active chlorine $\left(\mathrm{Cl}^{+}\right)$content on RCC-Cl was determined by standard iodometry/thiosulfate titration (Zhang et al. 2020). The calculation formula of effective $\mathrm{Cl}^{+} \%$ is as follows:

$\mathrm{Cl}^{+}(\%)=35.45 \times \mathrm{N} \times \mathrm{V} \times 100 / 2 \mathrm{~W}$

where $N$ and $V$ are the standard concentration (equivalent/liter) and volume (L) of the titrant (sodium thiosulfate), respectively, and $\mathrm{W}$ is the weight ( $\mathrm{g}$ ) of swatches used for each titration. It can be determined that active chlorine was not detected in the unchlorinated RCC as a control. 


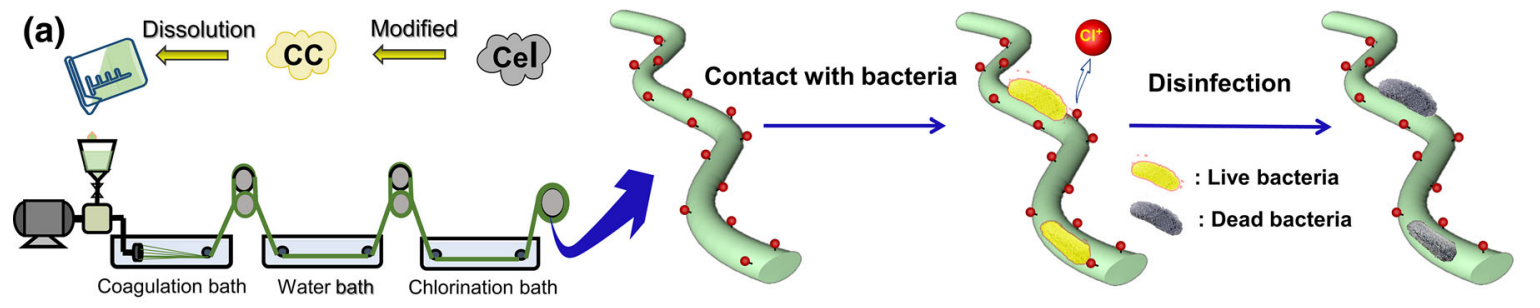

(b)

Wet spinning

RCC-Cl

Bacteria resisting Sterilized RCC-Cl

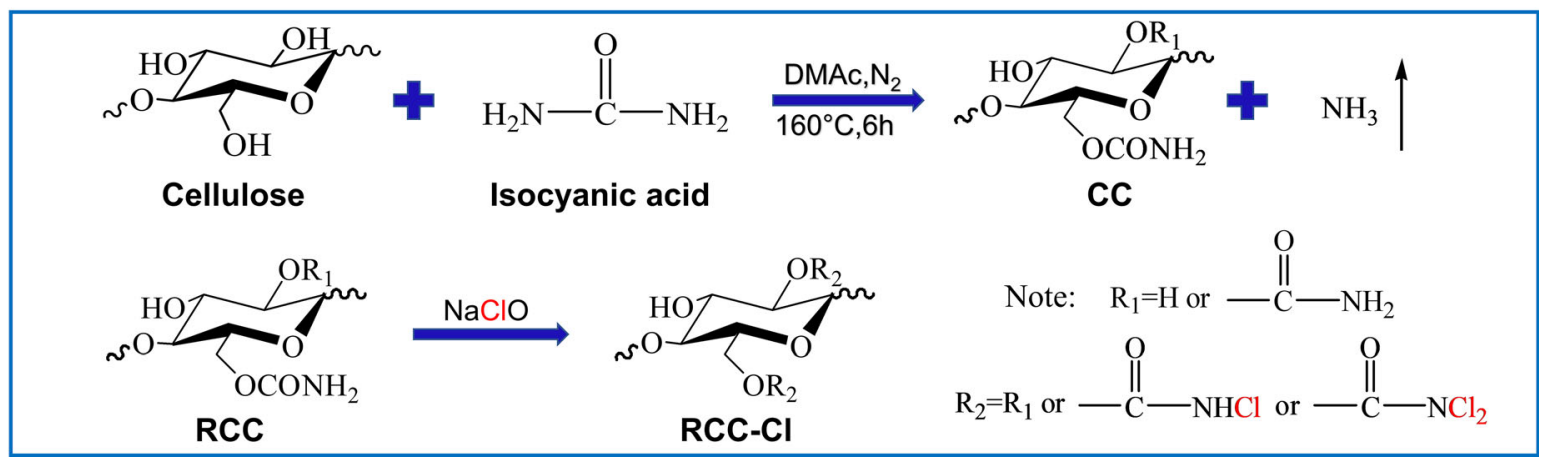

Fig. 1 a Schematic illustration of the design, preparation, and disinfection process of RCC-Cl. b Major synthesis pathways of RCC-Cl

\section{Stability and regenerability testing}

To study the stability of oxidative chlorine of RCC-Cl, the iodometric/thiosulfate titration method was used to periodically test the active chlorine content at room temperature for 35 days by storage in a dark environment.

For the rechargeability test, RCC-Cl carried out three chlorination-dechlorination cycles. Specifically, $\mathrm{RCC}-\mathrm{Cl}$ was chlorinated under optimal process conditions and washed with deionized water. Dried in an oven at $45^{\circ} \mathrm{C}$, the active chlorine content of RCC-Cl was determined by iodometry/thiosulfate titration. The RCC-Cl were exposed to $0.01 \mathrm{~N}$ sodium thiosulfate for $1 \mathrm{~h}$ to quench the chlorinated fibers. Then the fibers were washed with deionized water and chlorinated again.

\section{Antibacterial activity test}

Before conducting the antibacterial test, it was considered that there may be residual $\mathrm{NaClO}$ on the sample, which will interfere with the antibacterial effect. Therefore, a set of experiments was designed to exclude variables. RCC $(0.1 \mathrm{~g})$ was treated with $20 \mathrm{~g}$ of chlorinating solution. The chlorinated $\mathrm{RCC}-\mathrm{Cl}$ was washed with $20 \mathrm{~g}$ of deionized water each time and the solution after each wash was titrated. The modified AATCC test method 100-1999 was used to evaluate the antibacterial activity of chlorinated samples (Chen et al. 2020; Zhang et al. 2020). The unchlorinated and chlorinated fibers were attacked with $E$. coli (ATCC 25922) and S. aureus (ATCC 6538). In detail, $0.05 \mathrm{~g}$ of fiber was cut and pressed into a circular fiber mat with a radius of $2 \mathrm{~cm}$ (Fig. $2 \mathrm{e}_{2}, \mathrm{f}_{2}$ ). Then, $25 \mu \mathrm{L}$ of E. coli or $S$. aureus suspension containing approximately $10^{7} \mathrm{CFU} / \mathrm{ml}$ was added to the center of one fiber mat, and then another piece of the same fiber mat was placed on top of the first fiber mat. At the same time, the fiber mat was pressed by a sterile weight to ensure complete contact with bacteria. After 1, 5, 10, and $30 \mathrm{~min}$, the samples were quenched with $5 \mathrm{~mL}$ of $0.05 \mathrm{M}$ sodium thiosulfate solution to neutralize any residual chlorine oxide and terminate sterilization. The quenched samples were vortexed for $3 \mathrm{~min}$. After shaking was completed, the bacterial suspension was serially diluted with PBS, and then $25 \mu \mathrm{L}$ of the quenching solution was pipetted onto a nutrient agar culture plate and incubated at $37{ }^{\circ} \mathrm{C}$ for $24 \mathrm{~h}$. The antibacterial performance was evaluated by the colony counting method. 
(a)

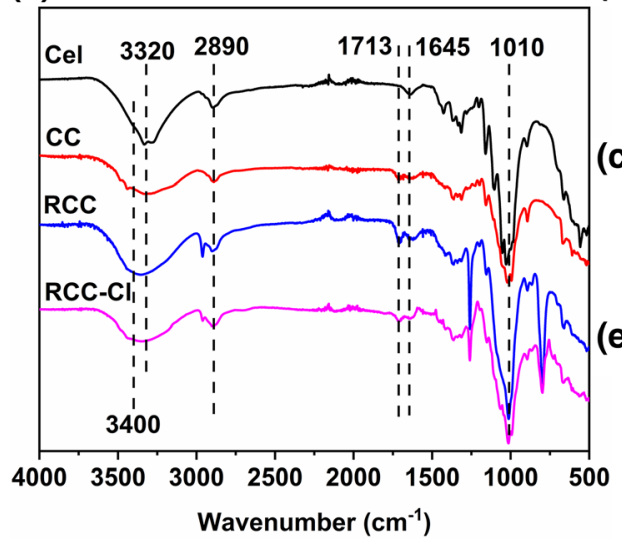

(b)

\begin{tabular}{ccccc}
\hline samples & Cel & CC & RCC & RCC-Cl \\
\hline $\mathbf{N} \%$ & 0 & 1.77 & 0.41 & 0.37 \\
\hline
\end{tabular}

(c) $\mathrm{Ce}$
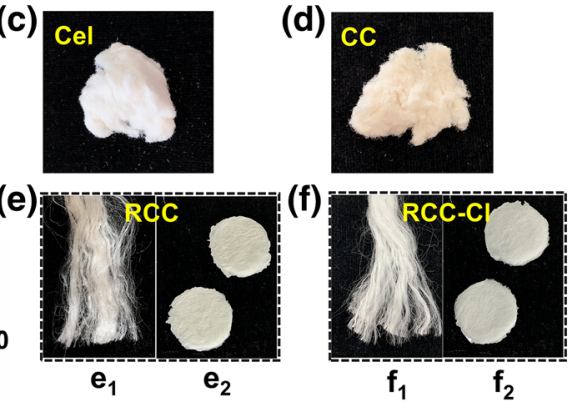

Fig. 2 FT-IR spectra (a), nitrogen content table (b) and actual photos of $\mathrm{Cel}(\mathbf{c}), \mathrm{CC}(\mathbf{d}), \mathrm{RCC}(\mathbf{e})$ and $\mathrm{RCC}-\mathrm{Cl}(\mathbf{f})$

\section{Results and discussion}

Chemical properties

\section{FT-IR analysis}

Figure 2a shows the FTIR spectrum of the test sample. The characteristic peaks at $3320 \mathrm{~cm}^{-1}, 1645 \mathrm{~cm}^{-1}$, and $1010 \mathrm{~cm}^{-1}$ of the sample are attributed to the bending vibration of $-\mathrm{OH}$ stretching (Zhang et al. $2019 b$ ). Furthermore, the absorption peak of the four curves at $2890 \mathrm{~cm}^{-1}$ can be regarded as an indication of the tensile vibration of the carbon-hydrogen bond in the sample (Xu et al. 2020). CC showed a new peak at $1713 \mathrm{~cm}^{-1}$, which was the contribution of the carbonyl group $(\mathrm{C}=\mathrm{O})$ in the carbamate, indicating that Cel has been successfully modified (Vo et al. 2010). The transmittance peaks at around $3400 \mathrm{~cm}^{-1}$ of CC and $\mathrm{RCC}$ showed the $-\mathrm{NH}_{2}$ functional groups, and no significant difference was found in the FTIR spectra of the two, indicating that dissolution regeneration would not completely change the chemical structure of carbamate (Menzel et al. 2017). After chlorination, no significant peak difference at $1713 \mathrm{~cm}^{-1}$ was found on RCC-Cl compared with RCC, indicating that chlorination did not destroy carbamate groups. To further determine the changes in carbamate groups in regenerated cellulose fibers, the samples were subjected to elemental analysis. It can be seen in Fig. $2 b$ that the modified cellulose had a lower degree of substitution, but nitrogen was still retained in the fiber after dissolution and regeneration, which indicates that the carbamate groups have been still retained in the regenerated cellulose fiber.

\section{Crystallization type analysis}

X-ray diffraction results showed the effects of esterification, dissolution, regeneration, and chlorination on the crystallinity of cellulose. The patterns of diffraction peaks in the XRD spectrum represent the crystalline state of the samples (French 2020). Figure 3 shows the XRD patterns of $\mathrm{Cel}, \mathrm{CC}, \mathrm{RCC}$, and RCC-Cl. The diffraction peaks of $\mathrm{Cel}$ and $\mathrm{CC}$ at $2 \theta$ values of $12.2^{\circ}, 20.3^{\circ}$, and $22^{\circ}$ are located on the $\left(1_{1}^{-} 0\right),(110)$, and $(020)$ planes, respectively, which are

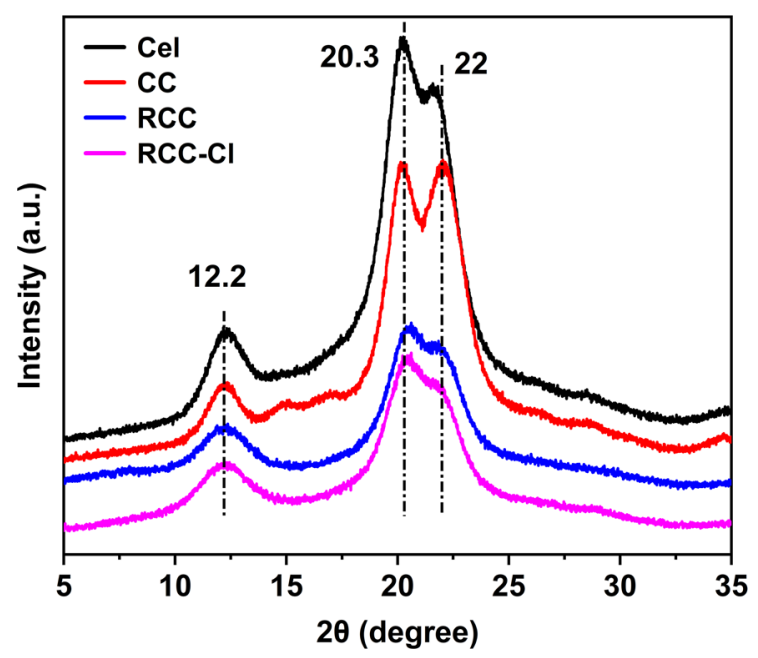

Fig. 3 X-ray diffraction patterns of a Cel, b CC, $\mathbf{c}$ RCC, and d $\mathrm{RCC}-\mathrm{Cl}$ 
the characteristic peaks of typical cellulose II (French 2014; Li et al. 2015). From the diffraction peak intensity, the crystallinity of CC was only marginally diminished from that of the Cel, indicating that the reaction was heterogeneous, which is also consistent with the lower nitrogen content of CC in Fig. 2b. After dissolving the regeneration of $\mathrm{RCC}$ and $\mathrm{RCC}-\mathrm{Cl}$, the diffraction peak intensity of cellulose type II was significantly reduced. This was because when the cellulose carbamate was dissolved in the $\mathrm{NaOH} /$ urea aqueous solution and regenerated, the cellulose hydrogen bond was destroyed and rebuilt, resulting in a decrease in crystallinity and orientation, so the regenerated fiber had a comfortable tactility and good elongation at break ( $\mathrm{Li}$ et al. 2015). In addition, the positions and intensities of the diffraction peaks of $\mathrm{RCC}$ and $\mathrm{RCC}-\mathrm{Cl}$ were the same, which indicated that the crystal form of cellulose did not change during the chlorination process.

\section{XPS analysis}

To further understand the microstructural changes in the material during the preparation of $\mathrm{RCC}-\mathrm{Cl}$, the surface chemical composition and the chemical state of Cel, CC, RCC, and RCC-Cl were studied by XPS. The low-resolution XPS spectrum is shown in Fig. 4. After modification of Cel, a characteristic peak at $398 \mathrm{eV}$ (N 1s) appeared in CC (Jiang et al. 2019). In addition, compared with the XPS spectrum of RCC, the obvious characteristic peak of $\mathrm{RCC}-\mathrm{Cl}$ was formed

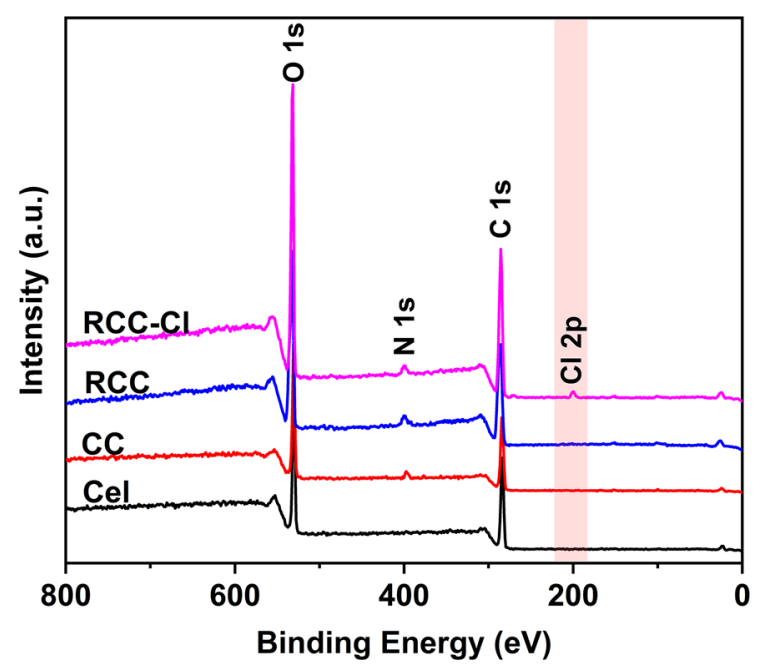

Fig. 4 XPS spectra of $\mathrm{Cel}, \mathrm{CC}, \mathrm{RCC}$, and RCC-Cl at $199.5 \mathrm{eV}(\mathrm{Cl} 2 p)$, indicating that chlorine had been successfully loaded onto the regenerated cellulose fiber (Liu et al. 2018).

\section{TG analysis}

The thermal degradation performance of Cel, CC, $\mathrm{RCC}$, and $\mathrm{RCC}-\mathrm{Cl}$ in nitrogen was assessed by TGA and DTG analysis. Figure 5 indicates a typical degradation of cellulose, divided into three weight loss steps. As shown in Fig. 5a, the first stage (in the region $25-140{ }^{\circ} \mathrm{C}$ ) showed the loss of moisture in the samples, which indicated that they had good water retention (Teng et al. 2018). Decomposition of the sample took place mainly in the second stage (within the range of $220-400{ }^{\circ} \mathrm{C}$ ), due to depolymerization of the cellulose, caused by breaking of $\beta(1 \rightarrow 4)$ glycosidic ether bonds, forming levoglucosan (1,6anhydro- $\beta$-d-glucopyranose) and carbon residues (Gorgieva et al. 2020). In the third stage, the residues of the sample slowly decreased with increasing temperature because the remaining residues were further oxidized. When the temperature reached $800{ }^{\circ} \mathrm{C}$, the residual amount of CC $(13.6 \%)$ was less than that of Cel $(16.9 \%)$, which was caused by the decrease in the crystallinity of cellulose after modification (Yu et al. 2016). The residual contents of RCC (24.5\%) and $\mathrm{RCC}-\mathrm{Cl}(28.2 \%)$ at $800{ }^{\circ} \mathrm{C}$ were higher than that of CC. This may be because part of the sodium atoms remained in the fiber during regeneration, which improved the carbonization ability of the fiber (Shi et al. 2017). In Fig. 5b, the DTG curve shows that both $\mathrm{Cel}$ and $\mathrm{CC}$ reached the maximum heat release rate at $350{ }^{\circ} \mathrm{C}$, showing two well-decomposed degradation peaks, whereas that of RCC and RCC-Cl reached their maximum heat release rate at $329{ }^{\circ} \mathrm{C}$ and $309{ }^{\circ} \mathrm{C}$, respectively. The relatively low temperatures at which $\mathrm{RCC}$ and $\mathrm{RCC}-\mathrm{Cl}$ achieved their maximum heat release rates may have been caused by a decrease in the crystallinity of the fiber after the cellulose was dissolved and regenerated, which was consistent with the XRD test results (Yu et al. 2016). 
(a) TG

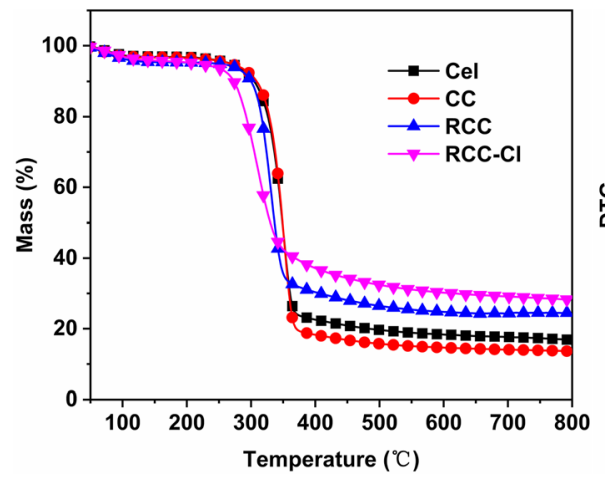

(b) DTG

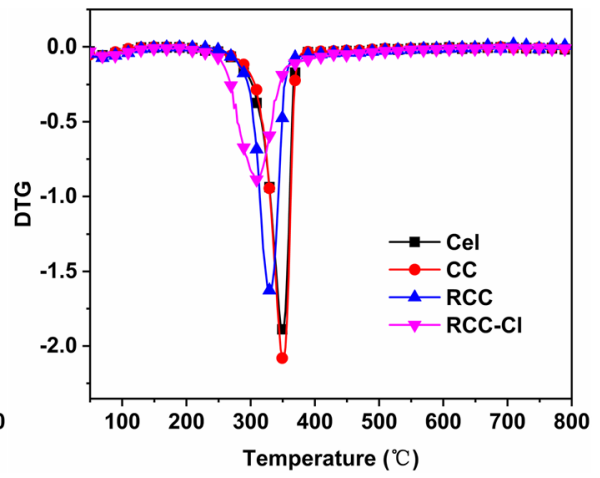

Fig. 5 TGA and DTG curves of a Cel, b CC, $\mathbf{c}$ RCC, and $\mathbf{d}$ RCC-Cl

Physical properties

\section{Mechanical property analysis}

Mechanical properties are important properties of fibers, and the mechanical properties of fibers are greatly affected by the spinning process. To understand the influence of the process conditions on the fiber properties, the mechanical properties of RCC fibers with various spinning solution concentrations, coagulation bath concentrations, and draw ratios were systematically studied. Figure 6a explores the effect of different spinning solution concentrations on the mechanical properties of the fiber. A spinning solution concentration of $6 \%$ can achieve a breaking strength of $12.4 \mathrm{cN} /$ tex and better break at elongation, while spinning at speeds that are too low or too high results in poor overall mechanical properties.

As shown in Fig. 6b, as the concentration of $\mathrm{H}_{2} \mathrm{SO}_{4}$ increased, the breaking strength of the fiber gradually increased. Under the effect of the same nozzle draw ratio, the coagulation and regeneration process of the fibers produced with a low concentration of $\mathrm{H}_{2} \mathrm{SO}_{4}$ was slow, which caused the phenomenon of merging and breaking, resulting in weak mechanical properties of the fibers (Ray et al. 2020). Under the action of $9 \%$ $\mathrm{H}_{2} \mathrm{SO}_{4}$, the process of fiber coagulation and regeneration is moderate, and the fiber with a breaking strength of $12.5 \mathrm{cN} /$ tex and a breaking elongation of $44.1 \%$ were showing good mechanical properties.

To further improve the mechanical properties of the as-spun fiber, it was necessary to change the draft ratio to draft the as-spun fiber. As shown in Fig. 6c, the asspun fiber was stretched in a constant temperature water bath at $25( \pm 2){ }^{\circ} \mathrm{C}$, the draw ratio were 1.1, 1.2, 1.3, and 1.4. The fiber fractured when the draw ratio was higher than 1.4, so the maximum draw ratio was limited to 1.4. With increasing draw ratio, the breaking strength of RCC increased and then decreased. The elongation at break of RCC decreased linearly with the increasing of the draft ratio. The maximum breaking strength of RCC reached $12.8 \mathrm{cN} /$ tex as the draw ratio increased to 1.2 times, and the breaking elongation reached $43.9 \%$.

After further optimization of the spinning process, special attention was given to the effect of chlorination treatment on fiber properties. Here, we compared the mechanical properties of RCC under the optimum process, $\mathrm{RCC}-\mathrm{Cl}$ after chlorination, $\mathrm{RCC}-\mathrm{Cl}\left(\mathrm{R}_{1}, \mathrm{R}_{2}\right.$, and $\mathrm{R}_{3}$ ) after three repeated quenching/recharging cycles, and RCC-Cl $\left(\mathrm{R}_{\mathrm{S}}\right)$ stored for more than 1 month (40-45 days). As shown in Fig. 6d, the fiber mechanical properties under the optimal process were verified. The breaking strength of RCC reached $12.8 \mathrm{cN} / \mathrm{tex}$, and the breaking strength of $\mathrm{RCC}-\mathrm{Cl}$ after chlorination was $12.1 \mathrm{cN} /$ tex, which was $5 \%$ lower than that of RCC. The elongation at break of RCC reached $44.5 \%$, and the elongation at break of RCC-Cl was $41.4 \%$, which was $7 \%$ lower than that of RCC. In general, the chlorination treatment did not significantly change the mechanical properties of the fiber. In addition, the mechanical properties of the three-cycle chlorinated RCC-Cl maintained a breaking strength of approximately $12 \mathrm{cN} /$ tex and a breaking elongation of approximately $37 \%$, which showed that the number of cycles did not significantly affect the mechanical properties of the fibers. Taking into account the strongly oxidizing properties of active chlorine, the 
(a)

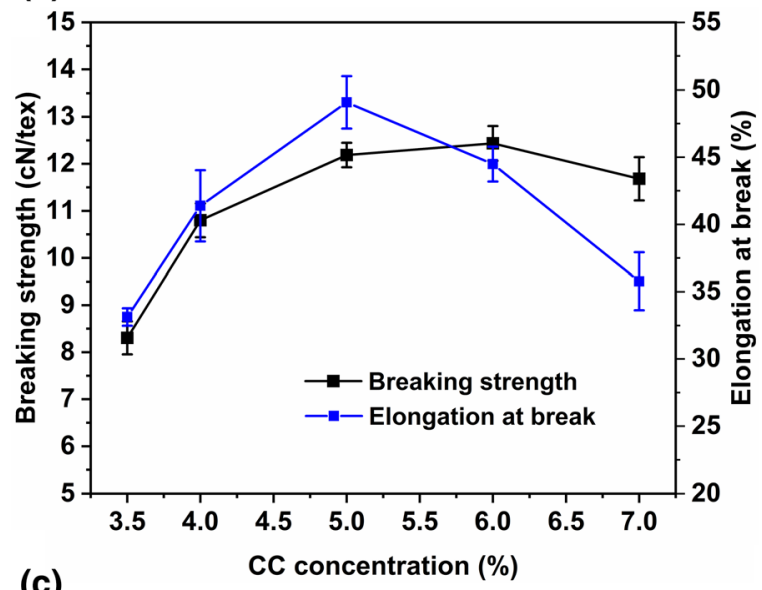

(c)

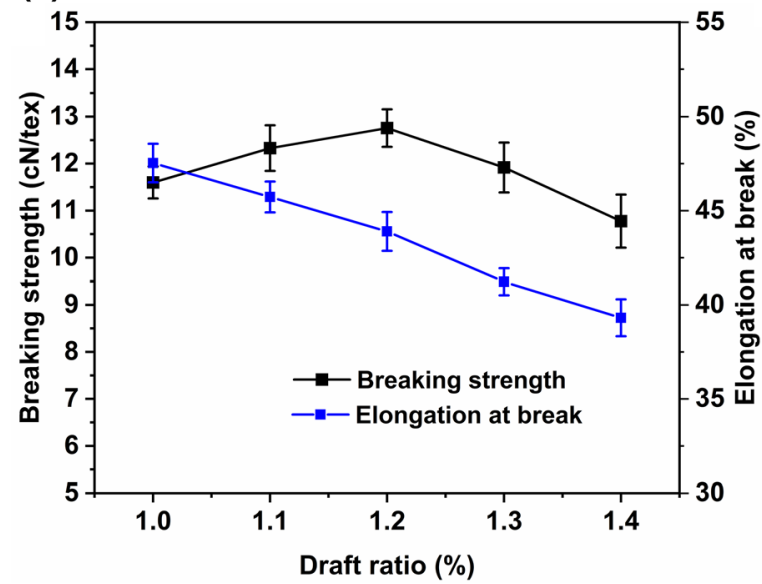

(b)
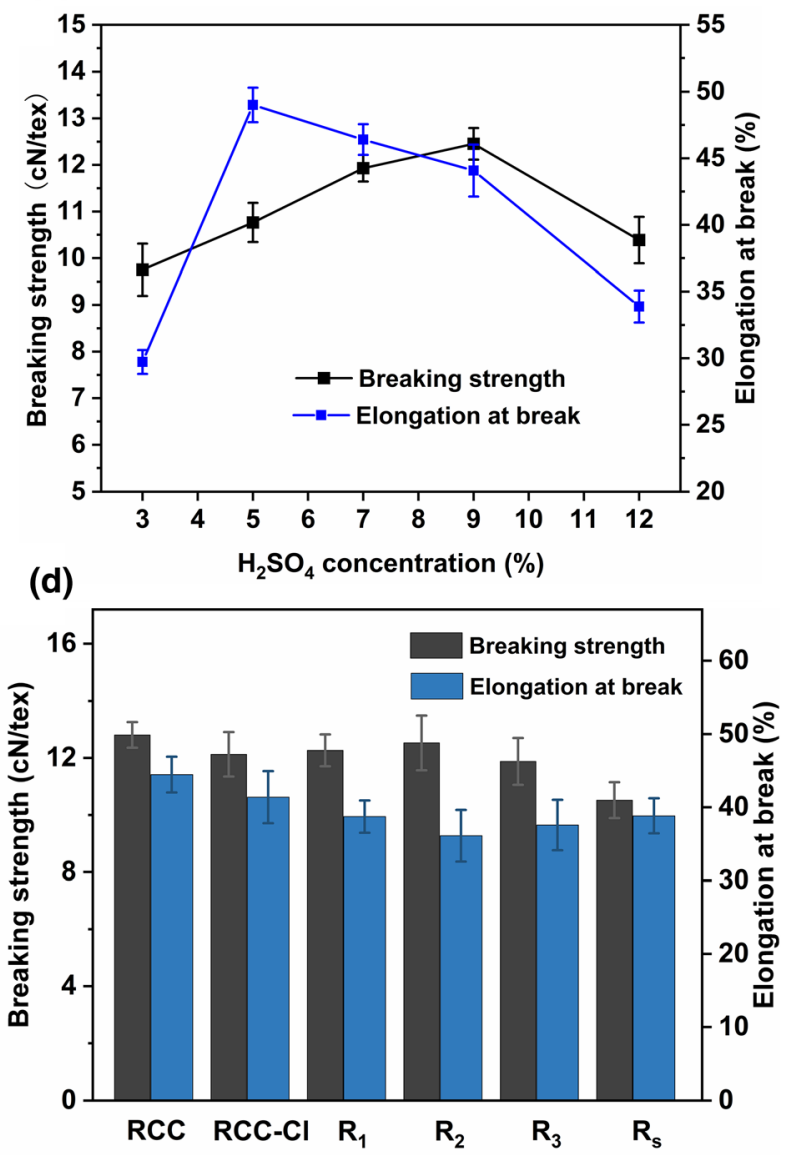

Fig. 6 The effects of different a spinning solution concentrations, $\mathbf{b}$ coagulation bath concentrations, $\mathbf{c}$ draw ratios and $\mathbf{d}$ chlorination treatments on the mechanical properties of the fibers

mechanical properties of $\mathrm{RCC}-\mathrm{Cl}$ stored for a long time were evaluated. The breaking strength of $R_{S}$ dropped to $10.52 \mathrm{cN} /$ tex, and the elongation at break was $38.84 \%$. The mechanical properties of the fibers slightly decreased after long-term storage.

\section{SEM-EDX analysis}

Figure 7 shows the SEM-EDX images of RCC fibers and RCC-Cl fibers. The RCC fiber exhibits a uniform and smooth surface, and the cross-section maintains a nearly circular/oval structure, as shown in Fig. 7a. When the fibers coagulated and formed, the colloid coagulated fully and uniformly, so that a homogeneous and compact structure was observed both in the outer layer and in the core. The fiber morphology of RCC-Cl (Fig. 7b) did not change significantly compared with that of RCC, indicating that the chlorination treatment did not change the surface/ cross-sectional structure of the fiber. This phenomenon was consistent with the good mechanical properties exhibited by both fibers. The EDX analysis of the RCC surface is shown in Fig. 7c. The elemental mapping image shows that nitrogen was evenly distributed on the surface of the fiber, which indicated that the low-substituted CC still retained a part of the amide bond after dissolution and regeneration. The EDX analysis of the RCC-Cl surface (Fig. 7d) shows a uniform distribution of chlorine in addition to nitrogen, which indicated that the chlorination treatment was successful. In addition, EDX analysis of the RCC and $\mathrm{RCC}-\mathrm{Cl}$ cross sections showed that sodium was indeed present in the fiber. 


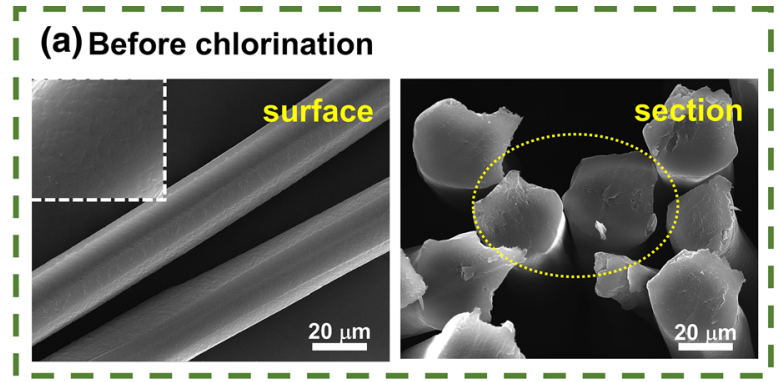

(c) Unchlorinated surface

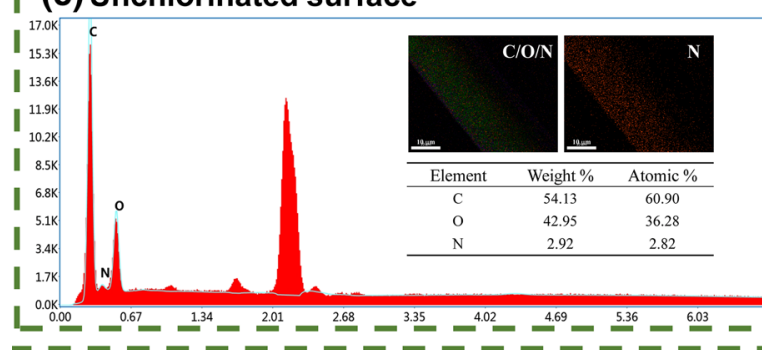

F (e) Unchlorinated section

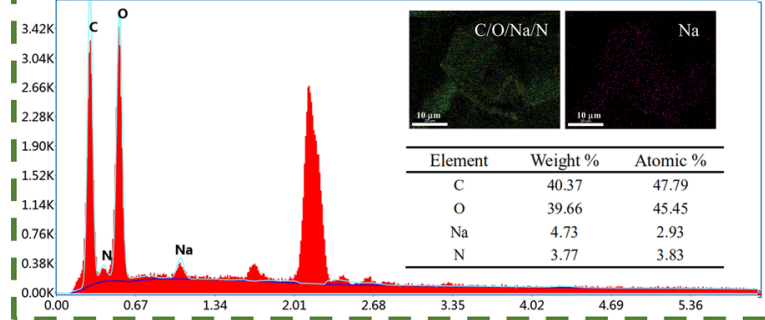

\section{(b) After chlorination}

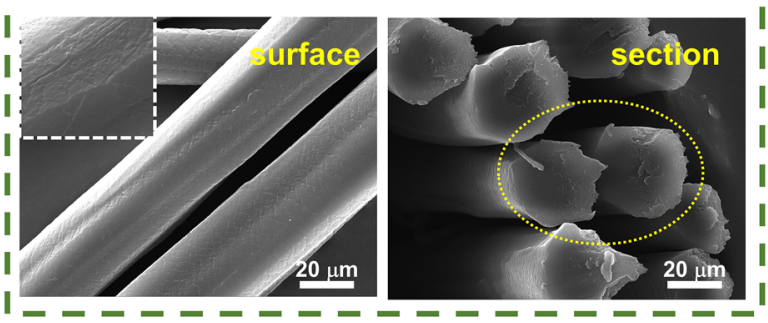

(d) Chlorinated surface

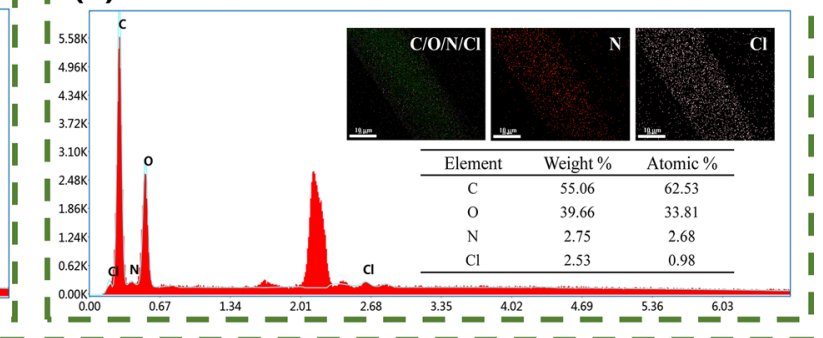

\section{(f) $\bar{C}$ horinated section}

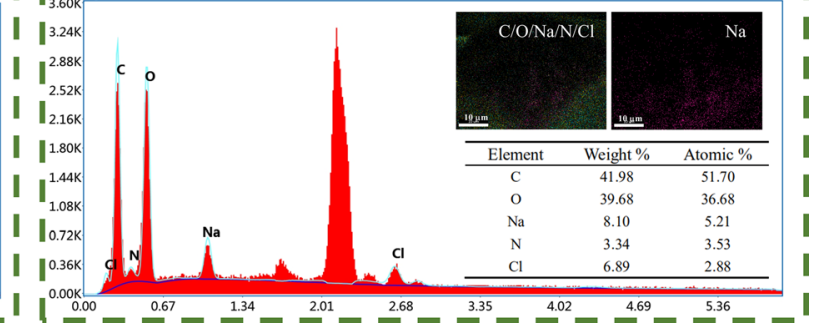

Fig. 7 SEM images $(\times 2000, \times 5000)$ of RCC $(\mathbf{a})$ and $\mathrm{RCC}-\mathrm{Cl}(\mathbf{b})$, and EDX mapping images and spectra $(\times 2000, \times 5000)$ of RCC (c, e) and RCC-Cl (d, f)

\section{Antibacterial properties}

\section{Effect of chlorination conditions}

$\mathrm{N}$-halamine is an organic compound containing one or more nitrogen-halogen covalent bonds, which are obtained by halogenation of $\mathrm{N}-\mathrm{H}$ groups. In inactivated microorganisms, N-halamine becomes $\mathrm{N}-\mathrm{H}$ bonds, and inactive $\mathrm{N}-\mathrm{H}$ bonds in dilute sodium hypochlorite solution can be reversed to $\mathrm{N}-\mathrm{X}$ bonds (Wang et al. 2020). Their antibacterial activity mainly depends on active chlorine, so the chlorination process of RCC should be optimized. The chlorinated solution was prepared using the available sodium hypochlorite solution, and the active chlorine content in $\mathrm{RCC}-\mathrm{Cl}$ was determined by iodometric titration. As shown in Fig. 8a, the chlorine content in the fiber increased sharply to $0.26 \%$ within $5 \mathrm{~min}$, and then slowly increased. After $15 \mathrm{~min}$ of chlorination, the saturated chlorine content was $0.27 \%$. In addition, the first derivative of the chlorine content curve was utilized to calculate the reaction rate of RCC-Cl. Compared with membranes and fabrics, the fiber had better hydrophilicity and a larger specific surface area. After the fibers came in contact with the chlorinated solution, the $\mathrm{N}-\mathrm{H}$ group was quickly converted to the $\mathrm{N}$-halamine group, and then the reaction rate gradually decreased to near zero after $5 \mathrm{~min}$. Therefore, it was determined that the saturation time of chlorination is about 5-10 min, and a chlorination time of 5 min was selected for subsequent experiments in consideration of industrial production efficiency. In this chlorination process, the chlorination effect mainly depends on the reaction between $\mathrm{HClO}$ and $-\mathrm{NH}_{2}$. The relative distribution of $\mathrm{Cl}_{2}, \mathrm{HClO}$, and $\mathrm{OCl}^{-}$in the chlorination solution is related to the $\mathrm{pH}$ value (Liu et al. 2018; Si et al. 2016). As shown in Fig. 8b, RCC-Cl showed a high chlorine content at $\mathrm{pH}$ 

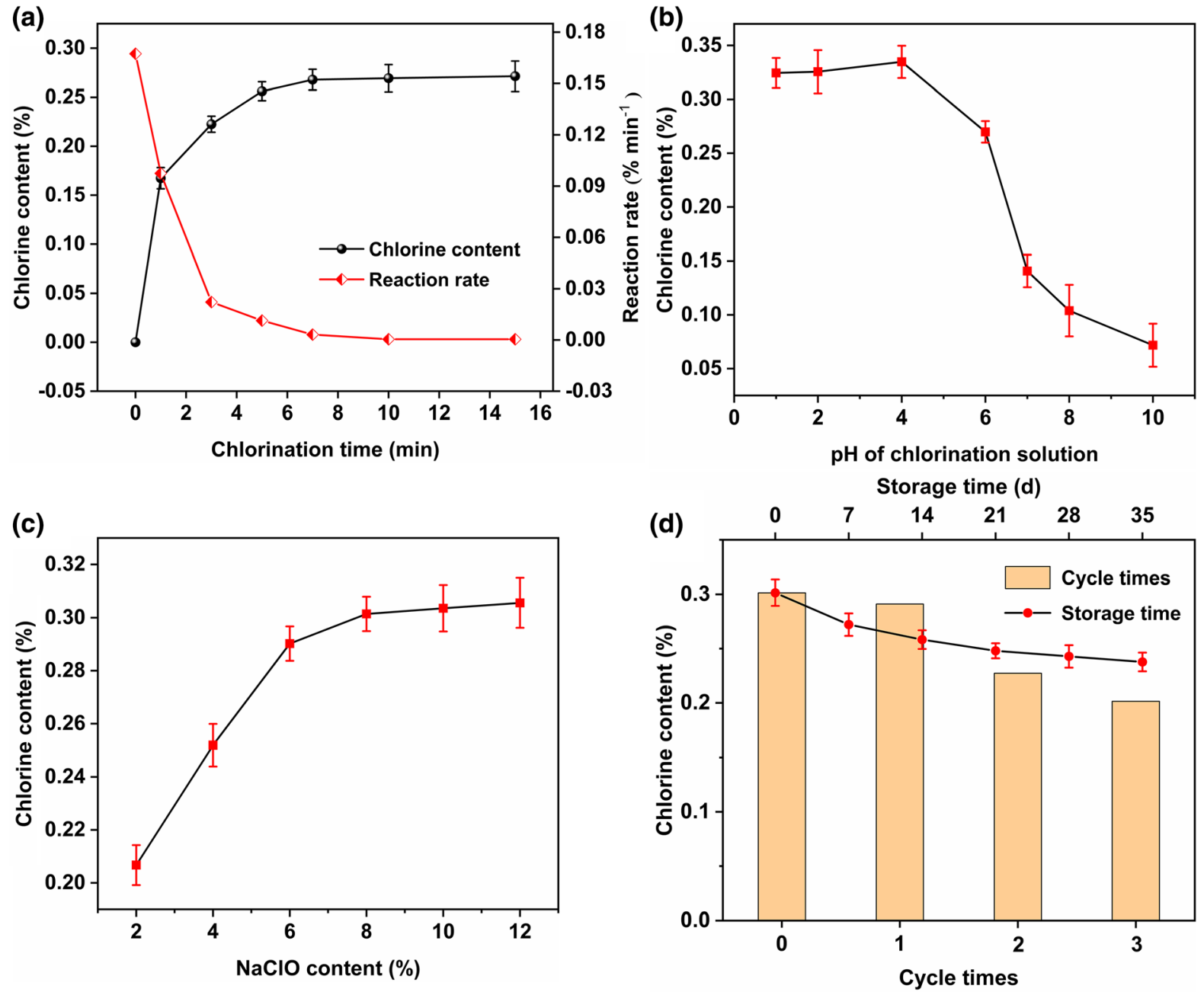

Fig. 8 Effect of a chlorination time, b solution $\mathrm{pH}$, and $\mathbf{c}$ sodium hypochlorite concentration of chlorination solution on the active chlorine content of RCC-Cl. d Change in active chlorine content with storage time and repeated chlorination times

values below 6 , which was attributed to the main form of chlorine being $\mathrm{HClO}$ ( $\mathrm{Si}$ et al. 2016). Therefore, the $\mathrm{pH}$ of the chlorinated solution was selected as 6 . Figure $8 \mathrm{c}$ explores the effect of the concentration of the chlorinated solution on the active chlorine content in RCC. The RCC was saturated at $8 \%$, so this concentration was selected as the concentration of the chlorinated solution. Under this process condition, the measured active chlorine content of $\mathrm{RCC}-\mathrm{Cl}$ was $0.3 \%$.

The durability of the antibacterial function of the fiber material is another issue to be considered. RCC$\mathrm{Cl}$ was stored in a dark place at room temperature $\left(25 \pm 2{ }^{\circ} \mathrm{C}\right)$ and the active chlorine content was measured intermittently. As shown in Fig. 8d, RCC-
$\mathrm{Cl}$ still retained nearly $80 \%$ of the original chlorine content after 35 days, highlighting the stability of the $\mathrm{N}$-halamine chemical structure in the fiber. To check this property, the active chlorine in $\mathrm{RCC}-\mathrm{Cl}$ was completely quenched with excess sodium thiosulfate solution, RCC-Cl was washed with deionized water and then chlorinated under the same chlorination conditions. The active chlorine content of RCC could still remain above $66 \%$ after 3 repeated quenching/ recharging cycles, and still maintained a good sterilization effect. 


\section{Antimicrobial efficacy test}

Before the antibacterial test, the RCC-Cl lotion in the washing process after chlorination was subjected to standard iodometry/thiosulfate titration, and color development images were collected. As shown in Fig. $9 \mathrm{a}, \mathrm{a}_{1}$ and $\mathrm{a}_{1 \mathrm{~S}}$ are the color development images of the chlorinated solution, and $\mathrm{a}_{2}, \mathrm{a}_{3}, \mathrm{a}_{4}, \mathrm{a}_{2 \mathrm{~S}}, \mathrm{a}_{3 \mathrm{~S}}$, and $\mathrm{a}_{4 \mathrm{~S}}$ are the color development images of the three washing solutions. It can be observed that the first lotion contained a large amount of $\mathrm{NaClO}$ solution, but $\mathrm{RCC}-\mathrm{Cl}$ contained almost no $\mathrm{NaClO}$ after two subsequent washes. The effective sterilization effect of RCC-Cl depended on its active chlorine.

The inactivation rates of different samples against E. coli and S. aureus were tested to characterize their antibacterial efficacy. The antibacterial performance was evaluated by the plate counting method, as shown in Fig. 9b, c. The two samples were loaded with E. coli $\left(4 \times 10^{7} \mathrm{CFU} \mathrm{mL} \mathrm{m}^{-1}\right)$ and $S$. aureus $\left(3.5 \times 10^{7}\right.$ $\mathrm{CFU} \mathrm{mL} \mathrm{m}^{-1}$ ) to observe the change in antibacterial effect with contact time. It can be observed that the RCC did not show a significant killing effect since only $\log$ reductions of 0.45 for $E$. coli and 0.54 for $S$. aureus were detected after $30 \mathrm{~min}$ of contact. The reason was that the bacteria adhered to the fiber surface instead of being inactivated. Because spherical $S$. aureus is more easily adhered to the fiber surface than rod-shaped E. coli, the reduction in S. aureus on RCC was more significant than that on E. coli (Jiang et al. 2014). In contrast, RCC-Cl had excellent killing activity against both strains, deactivating $100 \%$ of both $S$. aureus and E. coli within 1 min of contact. The fiber had a larger specific surface area than membranes and fabrics, and the evenly distributed active chlorine on the surface had an excellent bactericidal effect (Wang et al. 2020). Moreover, the manufacturing process of these antibacterial fiber $(\mathrm{RCC}-\mathrm{Cl})$ is

\section{(a)}

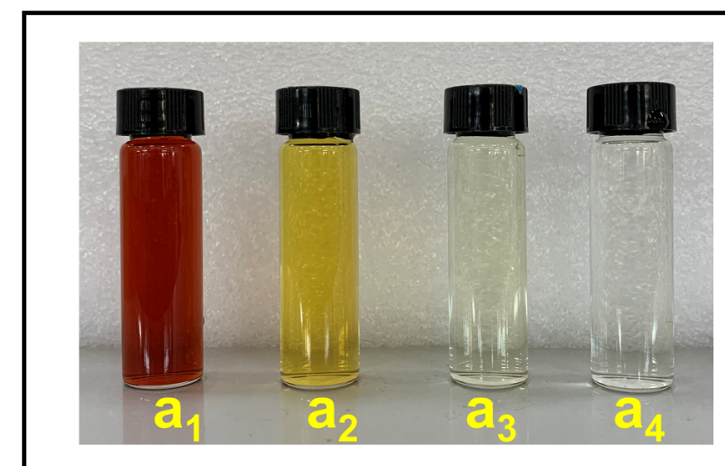

(b)

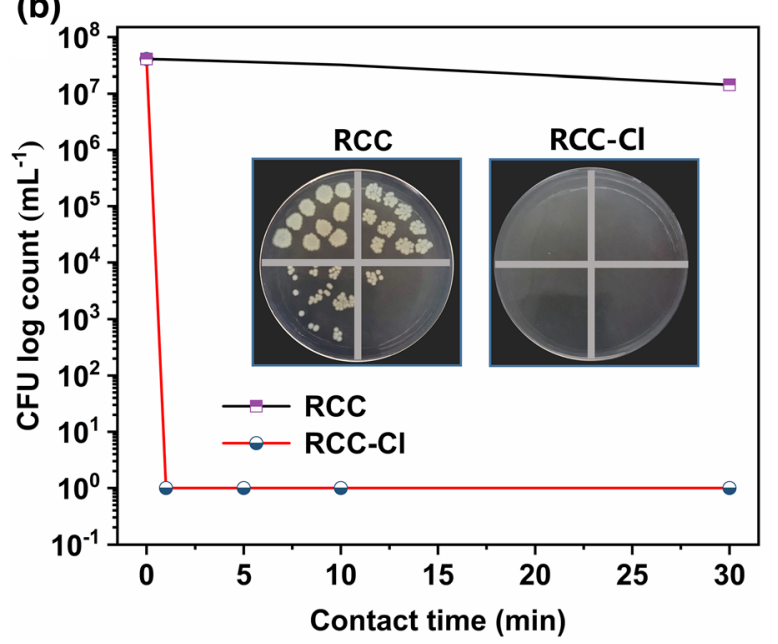

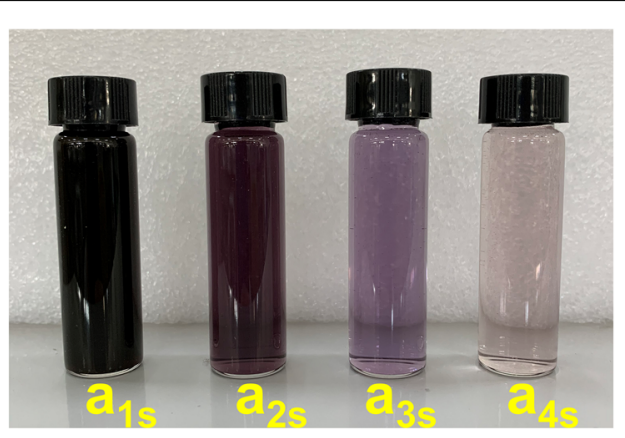

(c)

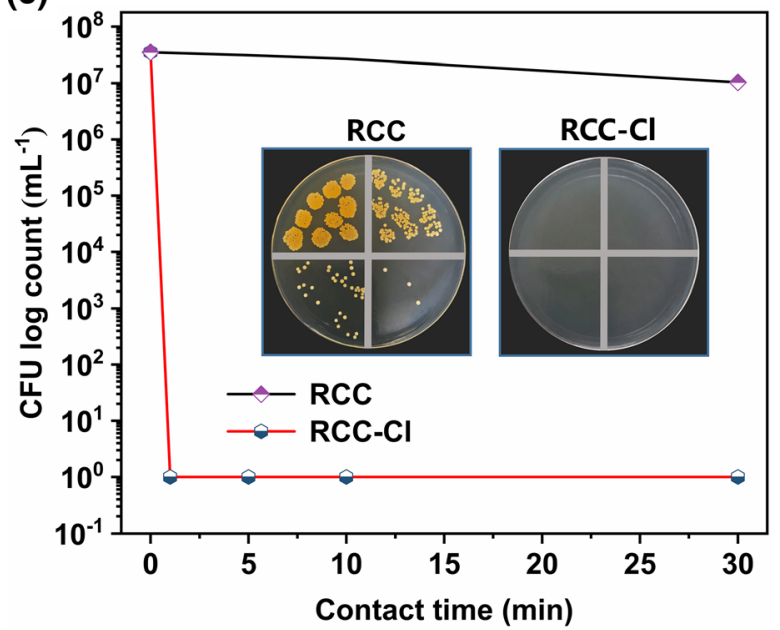

Fig. 9 Images a of color development by titration. Antimicrobial activities of RCC and RCC-Cl against b E. coli and c S. aureus. The images exhibit the plate-counting results after $1 \mathrm{~min}$ of exposure 
harmless to the environment, easy to prepare, low in cost, and shows great potential in the functional textile industry.

\section{Conclusion}

In this research, we proposed a fast and easy strategy to prepare regenerated cellulose fibers with excellent antibacterial effects by dissolving modified cellulose and the wet spinning technique. It was verified by FTIR and XPS spectra that cellulose was successfully grafted with amide groups, and amide groups remained on the regenerated cellulose fibers after being dissolved and wet-spun. Experimental results show that by optimizing the spinning process, thermally stable regenerated cellulose fiber (RCC-Cl) with a breaking strength of $12.1 \mathrm{cN} /$ tex and a breaking elongation of $41.4 \%$ can be obtained. The process of efficient chlorination to achieve $\mathrm{RCC}-\mathrm{Cl}$ storage stability, rechargeability, and high active chlorine content was explored. More importantly, RCC-Cl loaded with $0.3 \%$ active chlorine could inactivate E. coli and S. aureus at a concentration of $10^{7} \mathrm{CFU} /$ $\mathrm{mL}$ within $1 \mathrm{~min}$ after contact. Therefore, the prepared regenerated cellulose fibers have excellent antibacterial activity and might present potential applications in the functional textile field.

Funding This work was supported by State Key Laboratory of Bio-Fibers and Eco-Textiles (Qingdao University) (Grant Numbers No. ZKT43).

\section{Declarations}

Conflict of interest The authors declare that they have no known competing financial interests or personal relationships that could have appeared to influence the work reported in this paper. The manuscript was approved by all authors for publication.

Ethical approval This article does not contain any studies with human participants or animals performed by any of the authors.

Informed consent None.

\section{References}

Basu S, Omadjela O, Gaddes D, Tadigadapa S, Zimmer J, Catchmark JM (2016) Cellulose microfibril formation by surface-tethered cellulose synthase enzymes. ACS Nano 10:1896-1907. https://doi.org/10.1021/acsnano.5b05648

Bekri S et al (2020) New antibacterial cadiolide analogues active against antibiotic-resistant strains. Bioorg Med Chem Lett 30: https://doi.org/10.1016/j.bmcl.2020.127580

Chen J et al (2016) Novel PVDF hollow fiber ultrafiltration membranes with antibacterial and antifouling properties by embedding N-halamine functionalized multi-walled carbon nanotubes (MWNTs). RSC Adv 6:1710-1721

Chen Y, Wang Y, Feng C, He Q, Chen Q, Wang Z, Han Q (2020) Novel quat/di-N-halamines silane unit with enhanced synergism polymerized on cellulose for development of superior biocidability. Int J Biol Macromol 154:173-181. https://doi.org/10.1016/j.ijbiomac.2020.03.117

Coseri S (2017) Cellulose: to depolymerize... or not to? Biotechnol Adv 35:251-266. https://doi.org/10.1016/j. biotechadv.2017.01.002

Desai AN, Aronoff DM (2020) Masks and coronavirus disease 2019 (COVID-19). JAMA 323:2103-2103. https://doi.org/ 10.1001/jama.2020.6437

Elsayed S et al (2020) Recycling of superbase-based ionic liquid solvents for the production of textile-grade regenerated cellulose fibers in the lyocell process. ACS Sustain Chem Eng 8:14217-14227. https://doi.org/10.1021/ acssuschemeng.0c05330

French AD (2014) Idealized powder diffraction patterns for cellulose polymorphs. Cellulose 21:885-896. https://doi. org/10.1007/s10570-013-0030-4

French AD (2020) Increment in evolution of cellulose crystallinity analysis. Cellulose 27:5445-5448. https://doi.org/ 10.1007/s10570-020-03172-Z

Fu F, Yang Q, Zhou J, Hu H, Jia B, Zhang L (2014a) Structure and properties of regenerated cellulose filaments prepared from cellulose carbamate- $\mathrm{NaOH} / \mathrm{ZnO}$ aqueous solution. ACS Sustain Chem Eng 2:2604-2612. https://doi.org/10. $1021 / \mathrm{sc} 500559 \mathrm{~g}$

Fu F, Zhou J, Zhou X, Zhang L, Li D, Kondo T (2014b) Green method for production of cellulose multifilament from cellulose carbamate on a pilot scale. ACS Sustain Chem Eng 2:2363-2370. https://doi.org/10.1021/sc5003787

Fu F, Xu M, Wang H, Wang Y, Ge H, Zhou J (2015) Improved synthesis of cellulose carbamates with minimum urea based on an easy scale-up method. ACS Sustain Chem Eng 3:1510-1517. https://doi.org/10.1021/acssuschemeng. $5 \mathrm{~b} 00219$

Gorgieva S et al (2020) Processing and functional assessment of anisotropic cellulose nanofibril/Alolt/sodium silicate: based aerogels as flame retardant thermal insulators. Cellulose 27:1661-1683. https://doi.org/10.1007/s10570-01902901-3

Guo W, Liu W, Xu L, Feng P, Zhang Y, Yang W, Shuai C (2020) Halloysite nanotubes loaded with nano silver for the sustained-release of antibacterial polymer nanocomposite scaffolds. J Mater Sci Technol 46:237-247. https://doi.org/ 10.1016/j.jmst.2019.11.019

He L et al (2020) Using nano-selenium to combat coronavirus disease 2019 (COVID-19)? Nano Today. https://doi.org/ 10.1016/j.nantod.2020.101037

Jiang Z, Ma K, Du J, Li R, Ren X, Huang TS (2014) Synthesis of novel reactive $\mathrm{N}$-halamine precursors and application in 
antimicrobial cellulose. Appl Surf Sci 288:518-523. https://doi.org/10.1016/j.apsusc.2013.10.063

Jiang Z, Li H, He Y, Liu Y, Dong C, Zhu P (2019) Flame retardancy and thermal behavior of cotton fabrics based on a novel phosphorus-containing siloxane. Appl Surf Sci 479:765-775. https://doi.org/10.1016/j.apsusc.2019.02. 159

Kocer HB, Cerkez I, Worley SD, Broughton RM, Huang TS (2011) Cellulose/starch/HALS composite fibers extruded from an ionic liquid. Carbohydr Polym 86:922-927. https://doi.org/10.1016/j.carbpol.2011.05.046

Li J, Li R, Du J, Ren X, Worley SD, Huang TS (2013) Improved UV stability of antibacterial coatings with N-halamine/ TiO2. Cellulose 20:2151-2161. https://doi.org/10.1007/ s10570-013-9976-5

Li R, Wang S, Lu A, Zhang L (2015) Dissolution of cellulose from different sources in an $\mathrm{NaOH} /$ urea aqueous system at low temperature. Cellulose 22:339-349

Liu C, Shan H, Chen X, Si Y, Yin X, Yu J, Ding B (2018) Novel inorganic-based $\mathrm{N}$-halamine nanofibrous membranes as highly effective antibacterial agent for water disinfection. ACS Appl Mater Interfaces 10:44209-44215. https://doi. org/10.1021/acsami.8b18322

Liu W, Liu S, Liu T, Liu T, Zhang J, Liu H (2019) Eco-friendly post-consumer cotton waste recycling for regenerated cellulose fibers. Carbohydr Polym 206:141-148. https:// doi.org/10.1016/j.carbpol.2018.10.046

Menzel C, Seisenbaeva G, Agback P, Gällstedt M, Boldizar A, Koch K (2017) Wheat starch carbamate: production, molecular characterization, and film forming properties. Carbohydr Polym 172:365-373. https://doi.org/10.1016/j. carbpol.2017.05.053

Okugawa A, Sakaino M, Yuguchi Y, Yamane C (2020) Relaxation phenomenon and swelling behavior of regenerated cellulose fibers affected by water. Carbohydr Polym 231:115663. https://doi.org/10.1016/j.carbpol.2019. 115663

Pan N, Liu Y, Ren X, Huang T-S (2018) Fabrication of cotton fabrics through in-situ reduction of polymeric N-halamine modified graphene oxide with enhanced ultravioletblocking, self-cleaning, and highly efficient, and monitorable antibacterial properties. Colloid Surf A 555:765-771. https://doi.org/10.1016/j.colsurfa.2018.07. 056

Ray U, Zhu S, Pang Z, Li T (2020) Mechanics design in cellulose-enabled high-performance functional materials. Adv Mater. https://doi.org/10.1002/adma.202002504

Raza ZA, Abid S, Azam A, Rehman A (2020) Synthesis of alpha-tocopherol encapsulated chitosan nano-assemblies and their impregnation on cellulosic fabric for potential antibacterial and antioxidant cosmetotextiles. Cellulose 27(3):1717-1731

Sharma A, Sen D, Thakre S, Kumaraswamy G (2019) Characterizing microvoids in regenerated cellulose fibers obtained from viscose and lyocell processes. Macromolecules 52:3987-3994. https://doi.org/10.1021/acs.macromol. $9 \mathrm{~b} 00487$

Shi $\mathrm{R}$ et al (2017) Influence of $\mathrm{Na}^{+}$and $\mathrm{Ca}^{2+}$ on flame retardancy, thermal degradation, and pyrolysis behavior of cellulose fibers. Carbohydr Polym 157:1594-1603. https:// doi.org/10.1016/j.carbpol.2016.11.034
Si Y et al (2016) Mechanically robust and transparent N-halamine grafted PVA-co-PE films with renewable antimicrobial activity. Macromol Biosci 17:1600304

Sun X, Cao Z, Porteous N, Sun Y (2012) An N-halamine-based rechargeable antimicrobial and biofilm controlling polyurethane. Acta Biomater 8:1498-1506

Taheri H, Hietala M, Oksman K (2020) One-step twin-screw extrusion process of cellulose fibers and hydroxyethyl cellulose to produce fibrillated cellulose biocomposite. Cellulose 27:8105-8119. https://doi.org/10.1007/s10570020-03287-3

Teng Y, Yu GM, Fu YF, Yin CY (2018) The preparation and study of regenerated cellulose fibers by cellulose carbamate pathway. Int J Biol Macromol 107:383-392. https:// doi.org/10.1016/j.ijbiomac.2017.09.006

Vo LTT, Široká B, Manian AP, Bechtold T (2010) Functionalisation of cellulosic substrates by a facile solventless method of introducing carbamate groups. Carbohydr Polym 82:1191-1197. https://doi.org/10.1016/j.carbpol.2010.06.052

Wang F, Huang L, Zhang P, Si Y, Yu J, Ding B (2020) Antibacterial $\mathrm{N}$-halamine fibrous materials. Compos Commun 22:100487. https://doi.org/10.1016/j.coco.2020. 100487

Wu H et al (2018) Regenerated chitin fibers reinforced with bacterial cellulose nanocrystals as suture biomaterials. Carbohydr Polym 180:304-313. https://doi.org/10.1016/j. carbpol.2017.10.022

Xu M, Li T, Zhang S, Li W, He J, Yin C (2020) Preparation and characterization of cellulose carbamate membrane with high strength and transparency. J Appl Polym Sci. https:// doi.org/10.1002/app.50068

Yang Y, Zhang Y, Lang Y, Yu M (2018) Structure development in the condensed state of cellulose fiber regenerated from alkali complex solution. Cellulose 25:1555-1569. https:// doi.org/10.1007/s10570-018-1649-y

Yu G, Teng Y, Lai W, Yin C (2016) The preparation and study of cellulose carbamates and their regenerated membranes. Int J Biol Macromol 93:1155-1160. https://doi.org/10. 1016/j.ijbiomac.2016.09.081

Zahra H et al (2020) Close packing of cellulose and chitosan in regenerated cellulose fibers improves carbon yield and structural properties of respective carbon fibers. Biomacromol 21:4326-4335. https://doi.org/10.1021/acs. biomac.0c01117

Zhang S, Kai C, Liu B, Zhang S, Wei W, Xu X, Zhou Z (2019a) Preparation, characterization and antibacterial properties of cellulose membrane containing $\mathrm{N}$-halamine. Cellulose 26:5621-5633. https://doi.org/10.1007/s10570-019-02492-z

Zhang S, Yu C, Liu N, Teng Y, Yin C (2019b) Preparation of transparent anti-pollution cellulose carbamate regenerated cellulose membrane with high separation ability. Int J Biol Macromol 139:332-341

Zhang S, Li L, Ren X, Huang T-S (2020) N-halamine modified multiporous bacterial cellulose with enhanced antibacterial and hemostatic properties. Int J Biol Macromol 161:1070-1078. https://doi.org/10.1016/j.ijbiomac.2020.06.053

Publisher's Note Springer Nature remains neutral with regard to jurisdictional claims in published maps and institutional affiliations. 